\title{
CONSTIPAÇÃO INTESTINAL EM PACIENTES TRATADOS COM OPIOIDES: UMA REVISÃO INTEGRATIVA
}

\section{Constipation in patients treated with opioids: an integrative review \\ Estreñimiento en pacientes asistidos con opioides: una revisión integrativa}

\author{
Martiniano Bezerra de Lima \\ Escola de Saúde Pública do Estado do Ceará - ESP - CE - Fortaleza (CE) - Brasil
}

\section{Mayane Carneiro Alves Pereira}

Escola de Saúde Pública do Estado do Ceará - ESP - CE - Fortaleza (CE) - Brasil

\section{RESUMO}

Objetivo: Investigar na literatura o impacto da constipação intestinal induzida por opioides (CIO) por meio da identificação dos seus fatores de risco, sintomas e tratamentos. Métodos: Realizou-se, entre fevereiro e março de 2016, um levantamento de publicações científicas nas bases de dados eletrônicas Biblioteca Virtual em Saúde (BVS), Scientific Electronic Library Online (SciELO), Literatura Latino-Americana e do Caribe em Ciências da Saúde (LILACS) e Medical Literature Analysis and Retrieval System Online (MEDLINE), pesquisadas por meio dos descritores "analgésicos opioides", "constipação intestinal" e "dor", apresentados nos idiomas inglês, português e espanhol, correlacionados ou isolados, no período de 2011 a 2016, que investigassem pacientes em tratamento da dor com uso contínuo de medicamentos opioides e com desfecho clínico de constipação intestinal. Resultados: Os estudos apontaram a constipação intestinal como principal efeito secundário ao uso de opioides, os quais são muito importantes para o controle da dor de origem oncológica, assim como identificaram os fatores de risco para o surgimento da doença. Além disso, pacientes que foram bem assistidos por profissionais de saúde apresentaram melhor adesão ao tratamento da CIO. Conclusão: O controle da CIO proporciona conforto abdominal, autocuidado e redução nos custos do tratamento, ressaltando que deve haver capacitação dos profissionais de saúde, prevenção e acompanhamento periódico, além da precocidade dos tratamentos dietoterápico e medicamentoso.

Descritores: Analgésicos Opioides; Constipação intestinal; Dor; Neoplasias.

\section{ABSTRACT}

Objective: To investigate the impact of Opioid-Induced Constipation (OIC) in the literature through the identification of the risk factors, symptoms and treatments. Methods: We carried out a review of scientific publications available in the Virtual Health Library (VHL), the Scientific Electronic Library Online (SciELO), the Latin-American and Caribbean Center on Health Sciences Information (LILACS) and the Medical Literature Analysis and Retrieval System Online (MEDLINE) databases. The search was carried out using the descriptors "analgesic opioids", "constipation" and "pain" in English, Portuguese and Spanish, correlated or isolated. We searched for publications dating from 2011 to 2016 that investigated patients receiving treatment for pain with continuous use of opioids and with constipation as a clinical outcome. Results: The results highlighted constipation as the main secondary effect of opioids, which are very important for the control of cancer pain, and identified the risk factors for the disease. In addition, patients who were well served by health professionals presented better adherence to OIC treatment. Conclusion: The OIC control provides abdominal comfort, self-care and reduction in treatment costs. The importance of training health professionals and promoting prevention and periodic monitoring of patients should be highlighted. In addition, diet therapy and drug therapy should be provided early.

Descriptors: Analgesics, Opioid; Constipation; Pain; Neoplasms. 


\section{RESUMEN}

Objetivo: Investigar en la literatura el impacto del estreñimiento inducido por opioides (EIO) a través de la identificación de sus factores de riesgo, síntomas y tratamientos. Métodos: Se realizó entre febrero y marzo de 2016 una búsqueda de publicaciones científicas en las bases de datos electrónicas Biblioteca Virtual en Salud (BVS), Scientific Electronic Library Online (SciELO), Literatura Latino-Americana y del Caribe en Ciencias de la Salud (LILACS) y Medical Literature Analysis and Retrieval System Online (MEDLINE) investigadas a través de los descriptores "analgésicos opioides", "estreñimiento" y "dolor" en los idiomas inglés, portugués y español de manera individual o asociadas en el periodo entre 2011 y 2016 sobre investigaciones con pacientes en tratamiento del dolor en uso continuo de medicamentos opioides y desenlace clínico de estreñimiento. Resultados: Los estudios señalan el estreñimiento como principal efecto secundario del uso de opioides los cuales son muy importantes para el control del dolor de origen oncológico así como identificaron los factores de riesgo para el aparecimiento de la enfermedad. Además de eso, los pacientes que fueron bien asistidos por profesionales sanitarios presentaron más adhesión para el tratamiento del EIO. Conclusión: El control del EIO promueve el conforto del abdomen, el autocuidado y la reducción de los costes de tratamiento resaltando la necesidad de capacitación de los profesionales sanitarios, la prevención y el seguimiento periódico, además de la precocidad de los tratamientos de dieta y de medicamentos.

Descriptores: Analgésicos Opioides; Estreñimiento; Dolor; Neoplasias.

\section{INTRODUÇÃO}

Entende-se por opioides qualquer composto natural, semissintético ou sintético, que possua propriedades similares às dos opioides endógenos e que se liguem especificamente aos receptores opioides ( $m u$, kappa, delta e epsilon), que são importantes na regulação normal da sensação da dor ${ }^{(1)}$.

O ópio é uma substância original do grupo farmacológico dos opiáceos, extraído da papoula (Papaver somniferum). Historicamente, há mais de 4.000 anos se tem notícia do uso de opioides ${ }^{(2)}$. Em inúmeros eventos históricos são citados o uso do ópio, como na guerra do ópio, na China, onde sua utilização foi difundida por meio do fumo, e na guerra civil americana, havendo o emprego maciço do ópio, por via oral, e da morfina, por via subcutânea, nos soldados feridos em combate ou como forma de suportar as condições de batalha ${ }^{(3)}$.

Atualmente, os opioides são utilizados em situações de controle da dor em pacientes com dores neuropáticas ou mistas, lombalgias, cefaleias, nevralgias, artrite, insônia, inapetência, condições pré e pós-operatórias, anestesia, grandes queimaduras, politraumatizados $^{(4)} \mathrm{e}$ em pacientes oncológicos com ou sem possibilidade terapêutica ${ }^{(5)}$.

O alívio da dor em pacientes oncológicos requer o uso dessa classe de medicamentos para promover uma melhora na qualidade de vida desses indivíduos. Entretanto, a administração desses fármacos produz efeitos colaterais ${ }^{(6)}$, dentre os quais destaca-se a afecção do trato gastrintestinal, que pode provocar quadros de constipação intestinal ${ }^{(7)}$.

Em um estudo com pacientes que receberam opioides para tratamento paliativo da dor, observou-se que a constipação ocorreu em mais da metade dos pacientes, sendo quase sempre persistente, mesmo com o uso de laxativos ${ }^{(8)}$. De forma contrária, por meio de uma observação comum, em um ambulatório da dor de um hospital de referência no tratamento do câncer na região Norte/Nordeste, durante intervenções de médicos e residentes multiprofissionais à pacientes em uso de opioides, percebeu-se uma associação positiva entre a adesão aos tratamentos e a reabilitação intestinal. Desse modo, tal observação provocou a necessidade de entender a real dimensão da relação opioide-constipação intestinal. Assim, o presente estudo teve o objetivo de investigar na literatura o impacto da constipação intestinal induzida por opioides (CIO) por meio da identificação dos seus fatores de risco, sintomas e tratamentos.

\section{MÉTODOS}

O estudo proposto é uma revisão integrativa de literatura, que é um método para analisá-la de forma ampla, promovendo um embasamento teórico sólido, que pode contribuir para discussões sobre métodos e resultados de pesquisas, além de apontar lacunas do conhecimento que precisam ser preenchidas com novos estudos ${ }^{(9)}$.

Desse modo, foi realizado um levantamento de publicações científicas, entre fevereiro a março de 2016, nas bases de dados eletrônicas Biblioteca Virtual em Saúde (BVS), Scientific Electronic Library Online (SciELO), Literatura Latino-Americana e do Caribe em Ciências da Saúde (LILACS) e Medical Literature Analysis and Retrieval System Online (MEDLINE), pesquisadas por meio dos descritores "analgésicos opioides", "constipação intestinal" e "dor", apresentados nos idiomas inglês, português e espanhol, correlacionados ou isolados, no período de 2011 a 2016, que investigassem pacientes em tratamento da dor com uso contínuo de medicamentos opioides e com desfecho clínico de constipação intestinal. A questão norteadora da pesquisa foi: quão impactante é a interação medicamentosa de opioides sobre a constipação intestinal?

Como critérios de exclusão, foram rejeitados estudos realizados em animais, sem uso de opioides ou sem desfecho clínico de constipação intestinal, além de revisões bibliográficas, teses, dissertações, monografias e textos que estavam duplicados em mais de uma base de dados. 
A triagem se iniciou após a identificação dos artigos que contemplavam os descritores selecionados e que atendiam aos critérios de inclusão. Em seguida, foi realizada uma leitura seletiva dos artigos, partindo inicialmente da leitura dos títulos e resumos, para posterior análise exploratória, analítica e interpretativa dos artigos na íntegra, com a finalidade de tecer algumas considerações acerca do objeto de estudo desta pesquisa.

\section{RESULTADOS}

Inicialmente, foram encontrados 48 artigos, mas somente 10 contemplaram os critérios de elegibilidade. Os artigos científicos foram respectivamente organizados e descritos no quadro I conforme autor/ano, objetivo e principais resultados.

Dentre os artigos científicos selecionados, seis apresentaram-se em língua inglesa, dois na língua portuguesa e dois na língua espanhola. No que diz respeito aos objetivos dos artigos, $70 \%$ debatem questões ligadas aos sintomas da constipação, mas também abordam outras questões, como fatores de risco, tratamentos medicamentoso e dietoterápico, custos financeiros e as diferentes percepções do tratamento.

Quadro I - Descrição dos artigos de acordo com autor, ano, objetivo, população e resultados.

\begin{tabular}{|c|c|c|c|}
\hline Autor/Ano & Objetivo & População do estudo & Resultados \\
\hline $\begin{array}{l}\text { Dzierżanowski; } \\
\text { Ciałkowska-Rysz, } \\
2015^{(10)}\end{array}$ & $\begin{array}{l}\text { Avaliar a correlação entre a FdMI e } \\
\text { fatores de risco de constipação em } \\
\text { pacientes de cuidados paliativos. }\end{array}$ & $\begin{array}{l}\text { Pacientes em cuidados paliativos } \\
\text { atendidos em três centros } \\
\text { especializados submetidos a } \\
\text { questionários sobre sintomas de } \\
\text { disfunções intestinais, fatores de } \\
\text { risco comportamentais e uso de } \\
\text { opioides. }\end{array}$ & $\begin{array}{l}\text { Os principais fatores de risco de } \\
\text { constipação em pacientes de cuidados } \\
\text { paliativos foram: fluido insuficiente } \\
\text { e ingestão de alimentos, condições } \\
\text { inadequadas de privacidade, } \\
\text { dependência de um cuidador, bem como } \\
\text { o estado geral com desempenho ruim. }\end{array}$ \\
\hline $\begin{array}{l}\text { Chumpitaz- } \\
\text { Corredor; Lara- } \\
\text { Solares, 2012(11) }\end{array}$ & $\begin{array}{l}\text { Relatar o efeito da administração de } \\
\text { MTNX (12 mg) por via subcutânea } \\
\text { (SC) para aliviar a constipação } \\
\text { induzida por opioides. }\end{array}$ & $\begin{array}{l}\text { Pacientes terminais do INCMNSZ, } \\
\text { que foram avaliados durante a } \\
\text { admissão pela equipe clínica } \\
\text { da dor e cuidados paliativos e } \\
\text { que apresentavam constipação } \\
\text { secundária ao tratamento com } \\
\text { opioides. }\end{array}$ & $\begin{array}{l}\text { Todos os pacientes tiveram evacuação } \\
\text { após a aplicação da MTNX. Não houve } \\
\text { correlação entre a dose de opioide e } \\
\text { o tempo de resposta da MTNX. Não } \\
\text { houve correlação entre a dose de opioide } \\
\text { e os dias da constipação, ou entre dias } \\
\text { de constipação e resposta a MTNX. }\end{array}$ \\
\hline $\begin{array}{l}\text { Gálvez et al., } \\
2014^{(12)}\end{array}$ & $\begin{array}{l}\text { Analisar a prevalência e gravidade } \\
\text { de sintomas da DIO. }\end{array}$ & $\begin{array}{l}\text { Pacientes ambulatoriais com } \\
\text { diagnóstico de dor do câncer ou dor } \\
\text { não oncológica crônica tratados com } \\
\text { um único opioide. }\end{array}$ & $\begin{array}{l}\text { Pacientes tratados com opioide } \\
\text { apresentaram elevada frequência } \\
\text { de distúrbios gastrointestinais. A } \\
\text { constipação foi o sintoma mais comum, } \\
\text { ressaltando a necessidade de novas } \\
\text { estratégias para o tratamento. }\end{array}$ \\
\hline $\begin{array}{l}\text { Takemoto et al, } \\
2011^{(13)}\end{array}$ & $\begin{array}{l}\text { Estimar a prevalência de } \\
\text { constipação concomitante ao } \\
\text { tratamento com opioides e } \\
\text { comparar a utilização de recursos } \\
\text { e custos em pacientes tratados com } \\
\text { opioides com e sem constipação, } \\
\text { a partir da perspectiva do pagador } \\
\text { privado no Brasil. }\end{array}$ & $\begin{array}{l}\text { Pacientes com constipação intestinal } \\
\text { ou submetidos a procedimentos } \\
\text { relacionados durante o tratamento } \\
\text { com opioides, foram identificados } \\
\text { por um algoritmo em um banco de } \\
\text { dados longitudinal de planos de } \\
\text { saúde, divididos em quatro grupos: } \\
\text { G1- não tratados com opioides } \\
\text { sem constipação; G2- não tratados } \\
\text { com opioides com constipação; } \\
\text { G3- tratados com opioides sem } \\
\text { constipação; G4- tratados com } \\
\text { opioides com constipação. }\end{array}$ & $\begin{array}{l}\text { Pacientes com constipação intestinal } \\
\text { submetidos ao tratamento com opioides } \\
\text { exibiram um maior fardo econômico do } \\
\text { que os pacientes sem essa condição. }\end{array}$ \\
\hline
\end{tabular}




\begin{tabular}{|c|c|c|c|}
\hline $\begin{array}{l}\text { Laugsand et al, } \\
2011^{(14)}\end{array}$ & $\begin{array}{l}\text { Examinar a adequação do } \\
\text { tratamento para a constipação, } \\
\text { náuseas, depressão e falta de sono } \\
\text { e os fatores associados ao controle } \\
\text { dos sintomas inadequados em } \\
\text { pacientes com câncer submetidos } \\
\text { ao tratamento com opioides. }\end{array}$ & $\begin{array}{l}\text { Pacientes que receberam opioides } \\
\text { fortes para a dor oncológica, } \\
\text { recrutados de } 17 \text { centros em } 11 \\
\text { países europeus. }\end{array}$ & $\begin{array}{l}\text { A maioria dos pacientes com câncer } \\
\text { apresentavam tratamento inadequado, } \\
\text { ineficaz ou ausente. Houve subgrupos } \\
\text { de pacientes em risco particular } \\
\text { (constipação, náuseas, depressão e } \\
\text { sono de má qualidade) para tratamento } \\
\text { inadequado, que podem precisar } \\
\text { de atenção especial dos PCS para a } \\
\text { realização adequada do controle de } \\
\text { sintomas. }\end{array}$ \\
\hline Tai et al, 2016 $6^{(15)}$ & $\begin{array}{l}\text { Avaliar a gravidade dos sintomas } \\
\text { de pacientes com câncer avançado } \\
\text { em uma unidade de cuidados } \\
\text { paliativos e explorar os fatores } \\
\text { associados à melhora dos sintomas. }\end{array}$ & $\begin{array}{l}\text { Pacientes de uma unidade de } \\
\text { cuidados paliativos em Taiwan. }\end{array}$ & $\begin{array}{l}\text { A gravidade dos sintomas diminuiu } \\
\text { durante a primeira semana na unidade } \\
\text { de cuidados paliativos. Além disso, } \\
\text { as diferenças de sexo e locais de } \\
\text { câncer primário podem contribuir } \\
\text { para diferentes graus de melhora dos } \\
\text { sintomas. }\end{array}$ \\
\hline $\begin{array}{l}\text { Nunes; Garcia; } \\
\text { Sakata, 2014(16) }\end{array}$ & $\begin{array}{l}\text { Avaliar o uso de morfina como } \\
\text { primeiro medicamento para o } \\
\text { tratamento da dor oncológica } \\
\text { moderada, em pacientes com } \\
\text { doença avançada e/ou metástases, } \\
\text { como opção às recomendações da } \\
\text { escada analgésica preconizada pela } \\
\text { OMS. }\end{array}$ & $\begin{array}{l}\text { Pacientes com câncer, com idade } \\
\geq 18 \text { anos e sem uso de opioides. } \\
\text { Divisão em dois grupos: G1- } \\
\text { tratados com medicamentos segundo } \\
\text { a escada analgésica; G2 - tratados } \\
\text { com morfina. }\end{array}$ & $\begin{array}{l}\text { O uso de morfina como primeiro } \\
\text { medicamento para tratamento da dor } \\
\text { não promoveu melhor efeito analgésico } \\
\text { do que a escada preconizada pela OMS } \\
\text { e houve maior incidência de efeitos } \\
\text { adversos. }\end{array}$ \\
\hline $\begin{array}{l}\text { Marmo et al., } \\
2012^{(17)}\end{array}$ & $\begin{array}{l}\text { Avaliar o hábito intestinal de } \\
\text { pacientes com câncer em uso de } \\
\text { morfina. }\end{array}$ & $\begin{array}{l}\text { Pacientes } \\
\text { acompanhados no ambulatório de } \\
\text { dor do IOP da Universidade Federal } \\
\text { de São Paulo - SP. }\end{array}$ & $\begin{array}{l}\text { A constipação intestinal foi frequente, } \\
\text { entretanto, a atenção específica ao } \\
\text { hábito intestinal destes pacientes } \\
\text { aumentou a adesão aos laxantes e } \\
\text { reduziu a formação de fecaloma. }\end{array}$ \\
\hline $\begin{array}{l}\text { Feudtner et al., } \\
2014^{(18)}\end{array}$ & $\begin{array}{l}\text { Comparar pacientes que receberam } \\
\text { Senna com pacientes semelhantes } \\
\text { que receberam outras medicações } \\
\text { intestinal/oral, e determinar o } \\
\text { risco posterior de "constipação } \\
\text { problemática ". }\end{array}$ & $\begin{array}{l}\text { Pacientes com câncer, } \leq 20 \text { anos } \\
\text { de idade que, durante a internação, } \\
\text { foram expostos a sete ou mais dias } \\
\text { de uso consecutivos de opioides } \\
\text { e expostos à pelo menos um } \\
\text { medicamentor intestinal/oral } \\
\text { durante o } 1^{\circ} \text { ou } 2^{\circ} \text { dia do período de } \\
\text { referência. }\end{array}$ & $\begin{array}{l}\text { Iniciar a terapêutica com Senna, } \\
\text { em comparação com outros } \\
\text { medicamentos intestinal/oral, diminui } \\
\text { o risco subsequente de marcadores } \\
\text { de constipação problemática nesta } \\
\text { população. }\end{array}$ \\
\hline $\begin{array}{l}\text { Locasale et al., } \\
2016^{(19)}\end{array}$ & $\begin{array}{l}\text { Descrever a compreensão dos PCS } \\
\text { por meio do relato de experiências } \\
\text { dos pacientes com CIO. Avaliar } \\
\text { o grau de concordância ou } \\
\text { discordância de percepção entre } \\
\text { os pacientes e seus profissionais de } \\
\text { saúde que tratam a CIO e o impacto } \\
\text { sobre os resultados clínicos. }\end{array}$ & $\begin{array}{l}\text { Pacientes com idades entre } 18 \text { a } \\
85 \text { anos que receberam tratamento } \\
\text { com opioides diariamente por } \geq \\
4 \text { semanas para tratar a dor não } \\
\text { oncológica crônica com presença de } \\
\text { CIO. }\end{array}$ & $\begin{array}{l}\text { A importância e gravidade da CIO } \\
\text { são percebidos diferentemente pelos } \\
\text { pacientes e seus PCS, discordância que } \\
\text { complica o manejo da dor e demonstra a } \\
\text { necessidade de uma maior comunicação. }\end{array}$ \\
\hline
\end{tabular}

$\mathrm{CIO}=$ Constipação Induzida por Opioides; DIO = Disfunção intestinal Induzida por Opioides; FdMI = Frequência de Movimentos Intestinais; INCMNSZ = Instituto Nacional de Ciencias Médicas y Nutrición Salvador Zubirán; MTNX = Metilnaltrexona; IOP = Instituto de Oncologia Pediátrica; OMS = Organização Mundial da Saúde; PCS = Prestadores de Cuidados em Saúde.

\section{DISCUSSÃO}

Os resultados encontrados neste estudo apontaram a constipação intestinal como principal efeito secundário ao uso de opioides durante o tratamento da dor em todos os artigos analisados ${ }^{(10-19)}$. Em sete estudos a causa do uso de opioides estava ligado diretamente ao controle da dor com causa exclusivamente oncológica ${ }^{(10,11,14-18)}$, em outros dois a causa foi mista, 
oncológica/não-oncológica $^{(12-13)}$, e em apenas um foi exclusivamente não oncológica ${ }^{(19)}$. Além disso, a maioria da população estudada apresentou a média de idade maior ou igual a $60 \operatorname{anos}^{(10,11,14,15)}$, informação que parece sugerir uma correlação positiva entre câncer/idade avançada para o uso de opioides/CIO.

A CIO representa uma das causas do sofrimento não resolvido em pacientes durante o tratamento da dor. Normalmente, é pouco responsiva a laxantes regulares e pode ser um obstáculo à gestão eficaz da dor ${ }^{(10)}$. Além disso, ao contrário de outros efeitos colaterais, ela não desenvolve tolerância ao longo do tempo e aumenta com a duração do tratamento ${ }^{(10-12)}$, sendo o problema mais difícil durante o manejo da dor utilizando opioides ${ }^{(10)}$.

Um artigo apontou que pacientes relataram algum grau de impacto negativo dos efeitos gastrointestinais após seu uso em aspectos como trabalho, qualidade do sono, relações sociais, atividades diárias, qualidade de vida e estado de espírito em geral, e ainda destacou a constipação como a causa mais frequente para o abandono do tratamento a longo prazo ${ }^{(12)}$.

Do ponto de vista dos custos financeiros, observou-se em uma pesquisa que pacientes com CIO relataram expressivamente mais visitas ao médico e cuidados alternativos, além de serem mais susceptíveis de estarem em cuidados paliativos, o que favorece o aumento de gastos com o tratamento, reforçando o fato de que, comparativamente, a CIO tem maior influência sobre o uso de recursos e maiores custos do que pacientes sem essa condição ${ }^{(13)}$.

Para melhor entendimento da problemática dessa doença, a discussão desta pesquisa foi dividida em cinco eixos temáticos: sintomas e fatores de risco, pacientes com câncer, tratamento medicamentoso, tratamento dietoterápico e impacto dos profissionais de saúde sobre o tratamento.

\section{Sintomas e fatores de risco}

Três artigos definem a CIO como um sintoma originário na disfunção intestinal induzida por opioides (DIO), que é uma complicação comum no tratamento com opioides na dor crônica, cujos sintomas são caracterizados pela redução do esvaziamento gástrico, cólicas estomacais, edema, redução de movimentos intestinais e fezes excessivamente endurecidas ${ }^{(10-12)}$. Entre os sintomas, a constipação é o mais comum e debilitante da DIO, tendo a maioria das estimativas de prevalência baseadas apenas na frequência desse sintoma primário ${ }^{(12)}$.

Seis estudos relatam que, além da constipação intestinal, outros efeitos adversos, secundários a terapia da dor, comumente podem ocorrer e incluem: sonolência, náusea, vômito, acidez, flatulência, anorexia, distensão e dores abdominais ${ }^{(10-12,14-16)}$, que, por vezes, limitam o benefício do medicamento e podem provocar o abandono do tratamento ${ }^{(12)}$.

Quando administrado para analgesia da dor, os medicamentos opioides possuem afinidade pelos receptores $m u$, delta $e$ kappa, encarregados pela ação periférica e central dessas drogas. Entretanto apresentam problemas de seletividade, ocasionando efeitos adversos ao esperado, sendo o receptor mu o responsável pela $\mathrm{CIO}^{(12)}$. Ele possui ação central no intestino e, quando estimulado pela droga, influencia na inibição do peristaltismo intestinal, diminuindo a motilidade ${ }^{(11)}$.

Dois artigos sugerem que o diagnóstico da CIO deve basear-se em sintomas objetivos e subjetivos. Os sintomas objetivos, identificados por meio da frequência inferior a três evacuações por semana, e sintomas subjetivos, identificados pelo esforço de difícil eliminação, fezes excessivamente duras, com volume reduzido, sensação de evacuação incompleta, desconforto abdominal, plenitude gástrica e medidas facilitadoras para a saída do bolo fecal ${ }^{(10,12)}$.

Existem fatores de risco para o surgimento da CIO que dificultam o seu diagnóstico ${ }^{(14)}$. Quatro artigos atribuíram esses fatores a aspectos debilitantes na capacidade funcional dos pacientes, como: imobilização (especialmente em decúbito dorsal), baixa atividade física, idade avançada, aceitação insuficiente de líquidos e alimentos, doença oncológica abdominal, polimedicação, falta de privacidade, e instabilidade clínica e emocional ${ }^{(10,11,14,17)}$. Um estudo definiu que os fatores dependeram da presença de outros sintomas gastrointestinais, e esses, por sua vez, dependeram da duração da dor, sugerindo que provavelmente a incidência da CIO ocorre em pacientes com 12 meses ou mais em tratamento da dor crônica ${ }^{(12)}$.

Quando há a redução da capacidade funcional, os pacientes passam mais tempo acamados. Posteriormente, vai se reduzindo o apetite e a sede, de maneira que, juntamente com a progressão da doença, acabam tornando-se dependentes de seus cuidadores. Quando o quadro se intensifica, tornam-se mais suscetíveis a situações desconfortáveis para a defecação. Desse modo, já é possível antecipar o diagnóstico de constipação intestinal a partir da deterioração do estado de um paciente, o que é importante para a criação de ações profiláticas. Ressalta-se ainda que, independentemente do regime de cuidados, se hospitalar ou domiciliar, e da imobilidade, se completa ou parcial, (cadeirantes ou aqueles que não executam todos os movimentos), os pacientes são afetados por esses fatores de risco em um nível semelhante ${ }^{(10)}$.

A defecação é um direito básico à dignidade e honra dos pacientes, um ato íntimo que deve ser respeitado. Quando a privacidade não é garantida, os pacientes podem conter-se em relação aos movimentos intestinais, levando à constipação intestinal pelo adiamento da defecação. Essa condição normalmente ocorre entre pacientes hospitalares, sobretudo em cuidados paliativos, uma vez que são os mais prejudicados, devido à evacuação poder ocorrer de forma assistida pela equipe de saúde. É possível, em muitos casos, redimensionar o cuidado e criar um ambiente favorável, de modo a assegurar a privacidade desses pacientes $^{(10)}$. 


\section{Pacientes com câncer}

Tendo em vista que a maioria da população estudada nos artigos eram idosos e portadores de algum tipo de câncer ${ }^{(10,11,14,15)}$, é possível inferir que a idade pode apresentar-se como um fator de risco, tanto para a constipação quanto para o câncer. A doença, quando instalada, pode promover a dor oncológica e a busca para o controle da dor por meio do uso de opioides, os quais, consequentemente, podem ocasionar a constipação intestinal.

Mesmo a idade avançada se constituindo como um fator de risco para a CIO, o que não quer dizer que as crianças e adolescentes com câncer, tratadas com opioides, não estão imunes a essa condição, portanto, as recomendações de tratamento não devem ser extrapoladas para essa população, que requer tratamento diferenciado para o controle da doença, já que possuem características físicas, fisiológicas e psicológicas próprias ${ }^{(17)}$.

Os pacientes com câncer possuem uma situação clínica dinâmica, seja em relação à doença de base, seja em relação às comorbidades existentes, estando sujeitos aos mesmos fatores de riscos para a CIO já mencionados, dificultando o diagnóstico das possíveis causas da alteração do trânsito intestinal e consequente tratamento efetivo ${ }^{(17)}$. Todavia o controle dos sintomas é incentivado e deve ser tratado da forma mais precoce possível, em virtude da deterioração do estado funcional quando a constipação se estabelece ${ }^{(11)}$, além de permitir a compreensão do problema e melhorar a gestão clínica ${ }^{(12)}$.

Um estudo que analisou separadamente pacientes com câncer com e sem constipação, verificou que, comparativamente, pacientes com câncer e com constipação geram custos mais elevados de tratamento do que pacientes com câncer e sem constipação intestinal ${ }^{(13)}$.

No câncer avançado, as condições clínicas podem deteriorar-se de forma acentuada na fase terminal, resultando na piora repentina dos sintomas, assim a gestão rápida e eficaz desses sintomas é essencial para garantir conforto adequado e uma alta "qualidade de morte"(15).

\section{Tratamento medicamentoso}

Dois estudos afirmaram que os efeitos adversos da CIO seriam controlados com uso profilático de laxativos, sendo recomendada a prescrição de laxantes logo ao início do tratamento ${ }^{(16,17)}$. Outro estudo aponta que laxantes à base de Senna são bem tolerados em crianças e adultos, em cursos prolongados de terapia opioide, desde que não haja contraindicações, tendo baixo efeito colateral e baixo custo financeiro em relação a outros agentes farmacológicos e técnicas usadas para prevenir ou tratar a constipação intestinal, como enemas ou exposições a imagens abdominais ${ }^{(18)}$.

Três pesquisas consideram importante seguir as recomendações para o tratamento sintomático da CIO e individualizar a dose inicial, que deve ser baseada na intensidade da dor do paciente e na ocorrência de eventos adversos, podendo motivar a substituição de um opioide por outro. Essa individualização sugere que pode ser reduzida a incidência de efeitos adversos, refratários ou intoleráveis, e consequentemente melhorar a qualidade de vida dos pacientes ${ }^{(12,16,17)}$.

Nesse mesmo propósito, novos fármacos antagonistas de receptores opioides e com ação periférica, como oxicodona, naloxona e metilnaltrexona, mesmo possuindo mecanismo de ação idêntico ao opioides, acabam apresentando diferenças específicas na afinidade pelo receptor e farmacodinâmica, repercutindo em diferenças positivas na analgesia e na diminuição dos efeitos colaterais, podendo ser uma alternativa substitutiva a outros opioides ${ }^{(11,12,17)}$. Em dois estudos foi atribuída a essas drogas uma menor tendência à utilização de laxantes ${ }^{(11,17)}$.

Em casos de estabelecimento da impactação fecal, é indicada a realização de enemas, ainda que causem dor, desconforto e sejam de difícil manuseio, pois promovem o alívio temporário dos sintomas e conforto abdominal. Consiste na eliminação das fezes impactadas por meio de medicação por via retal ou com laxantes em altas doses por via oral. Entretanto, essa manobra é contraindicada em certas condições clínicas, como leucopenia, plaquetopenia, lesão anal ou imunodepressão grave, e sem o consentimento prévio do paciente ${ }^{(17)}$

\section{Tratamento dietoterápico}

Assim como a terapia medicamentosa pode ser essencial para o tratamento da constipação, de forma sinérgica, o incentivo à mudança dos hábitos alimentares, com maior ingestão de fibras e líquidos, também pode auxiliar nessa condição, uma vez que atuam diretamente sobre o funcionamento do intestino e a formação das fezes. No entanto, para atingir o sucesso no tratamento, as orientações dietéticas devem ser explicadas da forma mais clara possível para que os pacientes e/ou cuidadores minimizem os possíveis erros e obtenham sucesso no tratamento ${ }^{(10)}$.

É certo que, mesmo com o acompanhamento nutricional, oferta adequada de líquidos e alimentos, há casos difíceis ou impossíveis de serem revertidos devido a própria condição do paciente, como no caso de pacientes com caquexia, onde a ingestão pode ser insuficiente para o funcionamento normal do trânsito intestinal e consistência fecal. Nesses casos, o uso de suplementos nutricionais à base de fibras é contraindicado e, como prevenção eficaz da constipação intestinal, recomenda-se o aumento no fracionamento das refeições, com volume de líquidos e alimentos adequados à tolerância individual ${ }^{(10)}$.

\section{O impacto dos profissionais de saúde sobre o tratamento}

Apesar da incidência elevada, das dificuldades do tratamento e do aumento no número de pesquisas sobre CIO, há pouca melhoria no entendimento e comunicação dos profissionais de saúde sobre a intensidade de sintomas dos seus pacientes, 
gerando percepções diferentes sobre a importância e gravidade da doença entre os sujeitos ${ }^{(19)}$. Quatro estudos apontaram que a CIO e a dor crônica são pouco valorizadas ou subestimadas pelos profissionais da saúde e seus sintomas recebem uma atenção deficiente, constituindo-se como um tratamento inadequado ${ }^{(11,14,17,19)}$.

Um artigo evidenciou que a média de prescrição de opioides no Brasil está abaixo do consumo médio mundial, motivando uma evidência indireta do controle inadequado da dor no país ${ }^{(13)}$. Quando a constipação e a dor são subestimadas, cria-se uma barreira para a gestão dessas doenças ${ }^{(14)}$, podendo favorecer a presença de comportamentos negativos por parte do paciente, levando-o a suspender ou reduzir o consumo de opioides com a intenção de aliviar os sintomas de constipação; ou, inversamente, a dor gerada pelo desconforto da ausência de evacuações e pela distensão abdominal aumentar o consumo do medicamento, levando a um ciclo vicioso de intensificação da dor e da constipação, o que poderia levar até mesmo à criação de um estado de confusão aguda ${ }^{(11)}$. Por parte dos profissionais de saúde, tal condição pode contribuir para uma falta de consciência do impacto da $\mathrm{CIO}^{(19)}$.

As limitações na comunicação sobre os sintomas podem estar relacionadas a ambos os sujeitos. É evidenciada, entre os profissionais de saúde, a falta de tempo e iniciativa, por achar que os pacientes devem iniciar a conversação, ou pela limitação da abordagem de aspectos como sintomas, laxantes, satisfação, benefício e o impacto da CIO sobre o comprometimento da gestão da dor. Por parte dos pacientes, é observada a falta de clareza quanto ao nível dos sintomas ou omissão dos sintomas da CIO por diversas razões, como temor do não recebimento do opioide, não entendimento da relação opioide-constipação intestinal e crença de que constipação intestinal é uma condição de autocuidado, sendo, muitas vezes, considerada um constrangimento e permanecendo não compartilhada ${ }^{(19)}$.

Em outro estudo, observou-se que, no momento que os profissionais da saúde direcionam a atenção para diagnóstico, acompanhamento e tratamento da constipação intestinal, resulta numa melhor adesão aos laxantes recomendados, interferindo positivamente no manejo da doença ${ }^{(17)}$. Desse modo, para diminuir esse problema, devem ser promovidas educação clínica e coordenação dos cuidados voltados à gestão da CIO para os profissionais de saúde, além de um incentivo a um posicionamento mais proativo durante a prevenção, em que seja facilitada a comunicação sobre a constipação logo no início do tratamento com opioides entre os interessados, tornando os pacientes sujeitos comprometidos com a própria gestão da dor para evitar essa condição ${ }^{(19)}$.

\section{CONCLUSÃO}

Por meio desse estudo foi possível verificar o impacto da CIO em todos os seus aspectos: diagnóstico dos sintomas, fatores de risco ligados ao surgimento da doença, envolvimento no tratamento do dor oncológica, bem como as modalidades de tratamentos existentes. Além disso, demonstraram-se as discordâncias entre as percepções de pacientes e profissionais de saúde sobre o cuidado, mostrando que há falhas na comunicação que podem repercutir de forma negativa no tratamento da doença.

É importante que haja o manejo da CIO para proporcionar conforto abdominal e autocuidado nos pacientes com consequente redução nos custos do tratamento. Para tanto, faz-se necessário a capacitação dos profissionais de saúde, a prevenção para gestão de fatores de risco, o acompanhamento periódico, além da precocidade do tratamento dietoterápico (com orientações dietéticas e estímulo à mudança dos hábitos alimentares) e do medicamentoso (com prescrição de opioides ajustados de acordo com as alterações de dor ou efeitos adversos no paciente, e estímulo ao uso de laxantes).

\section{REFERÊNCIAS}

1. Baltieri DA, Strain EC, Dias JC, Scivoletto S, Malbergier A, Nicastri S, et al. Diretrizes para o tratamento de pacientes com síndrome de dependência de opióides no Brasil. Rev Bras Psiquiatr [Internet]. 2004 [acesso em 2016 Fev 18];26 (4):25969. Disponível em: http://dx.doi.org/10.1590/S1516-44462004000400011

2. Tanaka PP, Moss J. O papel dos antagonistas periféricos dos opióides no tratamento da dor e nos cuidados perioperatórios. Rev Bras Anestesiol [Internet]. 2008 [acesso em 2016 Fev 27]; 58(5): 533-547. Disponível em: http://dx.doi.org/10.1590/ S0034-70942008000500011

3. Duarte DF. Uma breve história do ópio e dos opióides. Rev Bras Anestesiol [Internet]. 2005 [acesso em 2016 Fev 29]; 55(1):135-46. Disponível em: http://dx.doi.org/10.1590/S0034-70942005000100015

4. Ministério da Saúde (BR), Instituto Nacional de Câncer. Cuidados paliativos oncológicos: controle da dor. Rio de Janeiro: INCA, 2001.

5. Caponero R, Jorge JMN, Melo AGC. Consenso brasileiro de constipação intestinal induzida por opióides. Rev Bras Cuidados Paliativos [Internet]. 2009 [acesso em 2016 Mar 03]; Supl 1:1-34. Disponível em: http://www.cuidadospaliativos. com.br/img/din/file/consenso_constip.pdf

6. Santos HS. Terapêutica nutricional para constipação intestinal em pacientes oncológicos com doença avançada em uso de opiáceos: revisão. Rev Bras Cancerol. 2002;48(2):263-9. 
7. Agra G, Fernandes MA, Platel ICS, Barros NCB, Freire MEM. Constipação em pacientes com doença oncológica avançada em uso de opioides. Mundo Saúde. 2013;37(4):472-8.

8. Kurz A, Sessler DI. Opioid-induced bowel dysfunction: pathophysiology and potential new therapies. Drugs. 2003;63:64971.

9. Mendes KDS, Silveira RCCP, Galvão CM. Revisão integrativa: método de pesquisa para a incorporação de evidências na saúde e na enfermagem. Texto \& Contexto Enferm [Internet]. 2008 [acesso em 2016 Fev 11];17(4):758-64. Disponível em: http://dx.doi.org/10.1590/S0104-07072008000400018

10. Dzierzanowski T, Ciałkowska-Rysz A. Behavioral risk factors of constipation in palliative care patients. Support Care Cancer [Internet]. 2015 [acesso em 2016 Fev 11];23:1787-93. Disponível em: https://www.ncbi.nlm.nih.gov/pmc/articles/ PMC4555193/pdf/520_2014_Article_2495.pdf

11. Chumpitaz-Corredor D, Lara-Solares A. Existe correlación entre la dosis de opioide y el tiempo de respuesta a metilnaltrexona. Rev Soc Esp Dolor [Internet]. 2012 [acesso em 2016 Mar 25];19(1):11-7. Disponível em: http://scielo. isciii.es/pdf/dolor/v19n1/original2.pdf

12. Gálvez R, Provencio M, Cobo M, Pérez C, Pérez C, Canal J. Prevalencia y severidad de la disfunción intestinal inducida por opioides. Atención Primaria [Internet]. 2014 [acesso em 2016 Mar 16];46(1):32-39. Disponível em: http://ac.els-cdn.com/S0212656713002722/1-s2.0-S0212656713002722-main.pdf?_tid=08f40dc8-9d7a-11e6-90a900000aab0f6c\&acdnat=1477705994_f81358dbe16d3aef24e279c3b53af3c0

13. Takemoto ML, Fernandes RA, Almeida GR, Monteiro RD, Colombini-Neto M, Bertola-Neto A. Health care resource use and costs in opioid-treated patients with and without constipation in Brazil. Value Health [Internet]. 2011 [acesso em 2016 Fev 15];14:78-81 Disponível em: http://www.ispor.org/consortiums/latinamerica/documents/ViH/Health-Care-ResourceUse-and-Costs.pdf

14. Laugsand EA, Jakobsen G, Kaasa S, Klepstad P. Inadequate symptom control in advanced cancer patients across Europe. Support. Care Cancer [Internet]. 2011 [acesso em 2016 Mar 30];19:2005-14. Disponível em: https://www.ncbi. nlm.nih.gov/pmc/articles/PMC3204099/pdf/520_2010_Article_1051.pdf

15. Shu-Yu T, Chung-Yin L, Chien-Yi W, Hui-Ya H, Joh-Jong H, Chia-Tsuan H, et al. Symptom patterns of advanced cancer patients in a palliative care unit: longitudinal assessments of symptoms improvement. BMC Palliat Care [Internet]. 2016 [acesso em 2016 Mar 30];15(1):32. Disponível em: https://www.researchgate.net/publication/297895780_Symptom_ severity_of_patients_with_advanced_cancer_in_palliative_care_unit_Longitudinal_assessments_of_symptoms_ improvement

16. Nunes BC, Garcia JBS, Sakata RK. Morfina como primeiro medicamento para tratamento da dor de câncer. Rev Bras Anestesiol [Internet]. 2014 [acesso em 2016 Mar 30];64(4):236-40. Disponível em: http://www.scielo.br/pdf/rba/v64n4/ pt_0034-7094-rba-64-04-00236.pdf

17. Marmo MCR, Caran EMM, Puty FCB, Morais MB. Avaliação do hábito intestinal em pacientes com câncer que utilizam morfina para o controle da dor. Rev Dor [Internet]. 2012 [acesso em 2016 Mar 16];13(3):243-8. Disponível em: http:// www.scielo.br/scielo.php?script=sci_arttext\&pid=S1806-00132012000300009\&lng=en\&nrm=iso

18. Feudtner C, Freedman J, Kang T, Womer JW, Dai D, Faerber J. Comparative effectiveness of senna to prevent problematic constipation in pediatric oncology patients receiving opioids: a multicenter study of clinically detailed administrative data. J Pain Symptom Manage [Internet]. 2014 [acesso em 2016 Mar 25];48(2):272-80. Disponível em: http://www.jpsmjournal. com/article/S0885-3924(13)00608-8/pdf

19. LoCasale RJ, Datto C, Wilson H, Yeomans K, Coyne KS. The burden of opioid-induced constipation: discordance between patient and health care provider reports. J Manag Care Spec Pharm [Internet]. 2016 [acesso em 2016 Mar 30];22(3):23645. Disponível em: http://www.jmcp.org/doi/pdf/10.18553/jmcp.2016.22.3.236

\section{Endereço para correspondência:}

Martiniano Bezerra de Lima

Escola de Saúde Pública

Avenida Antônio Justa, 3161

Bairro: Meireles

CEP: 60165-090 - Fortaleza - CE - Brasil

E-mail: soumartini@hotmail.com 